\title{
MATTHIEU PARIS, Le Moine et le Hasard. Bodleian Library, MS Ashmole 304
}

\section{Maria Colombo Timelli}

\section{OpenEdition}

\section{Journals}

\section{Édition électronique}

URL : https://journals.openedition.org/studifrancesi/12400

DOI : $10.4000 /$ studifrancesi. 12400

ISSN : 2427-5856

\section{Éditeur}

Rosenberg \& Sellier

\section{Édition imprimée}

Date de publication : 1 avril 2018

Pagination : 107

ISSN : 0039-2944

\section{Référence électronique}

Maria Colombo Timelli, « MatThieu PaRIs, Le Moine et le Hasard. Bodleian Library, MS Ashmole 304 », Studi Francesi [En ligne], 184 (LXII | I) | 2018, mis en ligne le 04 juillet 2018, consulté le 15 novembre 2021. URL : http://journals.openedition.org/studifrancesi/12400 ; DOI : https://doi.org/10.4000/ studifrancesi. 12400

Ce document a été généré automatiquement le 15 novembre 2021.

\section{(c) (i) (9)}

Studi Francesi è distribuita con Licenza Creative Commons Attribuzione - Non commerciale - Non opere derivate 4.0 Internazionale. 


\title{
MATTHIEU PARIS, Le Moine et le Hasard. Bodleian Library, MS Ashmole 304
}

\author{
Maria Colombo Timelli
}

\section{RÉFÉRENCE}

MATTHIEU PARIS, Le Moine et le Hasard. Bodleian Library, MS Ashmole 304. Texte présenté par Allegra Iafrate, Paris, Classiques Garnier, 2015, « Textes littéraires du Moyen Âge» 39, «Divinatoria» 5, 277 pp.

1 Ce volume propose la reproduction en fac-similé et en couleur du ms Oxford, Bod. Lib., Ashmole 304 ; bien que les textes qu'il contient soient tous en latin, l'importante introduction d'A.I. pourra intéresser nos lecteurs, dans la mesure où elle fait utilement le point sur la vie et l'œuvre de Matthieu Paris (ca 1200-1259), moine bénédictin dans l'abbaye Saint-Albans près de Londres, connu en tant que chroniqueur, diplomate, copiste et enlumineur, qui n'a pas négligé d'utiliser la langue vulgaire - l'anglonormand en l'occurrence - pour ses œuvres hagiographiques. A.I. présente d'abord l'ensemble de sa production (au double sens du terme : composition de textes et facture des manuscrits, pp.12-20), avant de se concentrer longuement sur les textes divinatoires transmis par le ms d'Oxford (pp. 20-88) et d'offrir une description codicologique détaillée (pp. 89-103). Bibliographie aux pp. 253-266.

2 Précieux recueil, quoique nécessairement partiel, rendant compte de l'ensemble de la production d'une des plus grandes spécialistes de la littérature médiévale, ce gros volume a plusieurs mérites.

3 Le premier est de réunir des contributions jusqu'ici relativement dispersées, souvent parues dans des volumes difficilement accessibles en dehors des grandes bibliothèques. Les intitulés des cinq parties donnent la mesure de la cohérence, de la continuité, mais aussi de la variété, du corpus : la célèbre synthèse sur La littérature religieuse parue dans le Grundriss der romanischen Literaturen des Mittelalters (1988) constitue à elle seule la première section, les quatre autres portant sur les lectures spirituelles ( 9 articles : la 
présence de la table des matières en-ligne sur le site de Brepols nous dispense d'en donner la liste), les traductions (4 articles), la prédication et la pastorale (6 articles), auxquelles s'ajoute une partie plus hétérogène centrée sur les pratiques des copistes et sur le vocabulaire ( 8 articles). Sans revenir ici sur le détail, on soulignera au moins l'approche globale de Geneviève Hasenohr, qui allie à l'étude des textes celle de leur tradition, les questions linguistiques et souvent l'histoire de la transmission des œuvres au sein des collections anciennes.

Deuxième mérite, absolument novateur dans des recueils de ce genre : les vingt-sept contributions réunies ici ont été soigneusement revues, non pas à l'intérieur du texte (cela aurait amené, sinon à les récrire partiellement, à compléter voire à refaire les notes), mais par quelques lignes, ou quelques pages, de remarques qui constituent autant de post-scriptum critiques : c'est là que le lecteur trouvera des ajustements, des renvois bibliographiques récents, parfois objets de discussion, ainsi que des remarques ponctuelles. Il est néanmoins remarquable que la mise à jour de quelques points de détail ne remet de toute façon jamais en cause la validité des panoramiques proposés : preuve de la modernité et de la valeur des articles (dont le plus ancien date de 1978, le plus récent de 2007) et de la capacité de leur auteur d'avoir une perception d'ensemble des phénomènes appuyée sur des informations fondées et approfondies que les progrès de la critique n'ont pas remise en cause.

Le troisième mérite revient aux anciennes collègues, amies et élèves de $G$. Hasenohr qui ont codirigé ce recueil - Marie-Clotilde Hubert, Sylvie Lefèvre, Anne-Françoise Leurquin, Christine Ruby, Marie-Laure Savoye - en le complétant par une bibliographie exhaustive (pp. 17-24) et surtout par des Indices (auteurs et œuvres; personnes, lieux et milieux; manuscrits) qui révèlent certes des parcours, des sujets et des auteurs privilégiés, mais permettent surtout le repérage rapide des informations au lecteur intéressé à des recherches ponctuelles.

6 Soulignons enfin qu'un article apparaît ici en traduction française (Les lectures religieuses des laïcs dans la France du Xve siècle : norme et pratique, lumières et ombres, 1994, version originale en anglais) et surtout que la très précieuse contribution sur $L a$ littérature religieuse à laquelle on a fait allusion (GRLMA VIII/1, 1988) est ici complétée par les « Fiches» qui auraient dû trouver place dans le volume VIII/2, jamais paru; ces quelque 180 notices (pp.79-143) ajoutent aux informations principales sur chaque œuvre, à la bibliographie de base et à la liste des manuscrits, le recensement des éditions anciennes: bien que basé sur les catalogues disponibles (Gesamtkatalog der Wiegendrucke, Universal Short Title Catalogue, French Vernacular Books), dont les données n'ont pas pu être toutes vérifiées, celui-ci représente une nouveauté par rapport aux critères du GRLMA et un véritable enrichissement, dans la mesure où il intègre les perspectives les plus récentes de la recherche, en dépassant les prétendues frontières entre Moyen Âge et Renaissance, transmission manuscrite et imprimée, pour essayer de mieux cerner la réception même des textes.

7 Ces compléments rendent ce volume bien plus riche qu'un simple recueil « d'auteur», et le transforment en une sorte de petite bibliothèque à laquelle beaucoup de médiévistes, jeunes et moins jeunes, pourront puiser de précieuses ressources. 